\title{
Some Proposition that Links Ferromagnetic Models with Cantorian Set Theory
}

\author{
Mohamed Atef Mohamed Gebril ${ }^{1}$ \\ ${ }^{1}$ Department of material engineering, Faculty of petroleum and minning engineering, Suez University, Egypt \\ Correspondence: Mohamed Atef Mohamed Gebril, Department of material engineering, Faculty of petroleum and \\ minning engineering, Suez University, Egypt. E-mail: atefmohamed840@yahoo.com
}

Received: July 13, 2015

Accepted: March 2, 2016

Online Published: October 21, 2016

doi:10.5539/apr.v8n6p1

URL: http://dx.doi.org/10.5539/apr.v8n6p1

\begin{abstract}
In this paper, we established a link between ferromagnetic models and set theory. Three dimension spins " $\mathrm{S}_{\mathrm{X}}, \mathrm{S}_{\mathrm{y}}$, $\mathrm{S}_{\mathrm{z}}$ " are considered in Heisenberg model but it is restricted to $\mathrm{z}_{-}$axis " $\mathrm{S}_{\mathrm{z}}$ " in Ising mode. By using Brouwer theory, fractal motion of magnetic domains is predicted theoretically in the materials that their magnetism are explained by Ising model. In addition, we achieved that the experimental data of the fractal motion of magnetic domains in the thin films are agreement with the theoretical assumptions that are proposed.

Keywords: Heisenberg model, Ising model, Ginzburg-Landau equations, ZFC, Large Cardinals, Fractals, Space filling curve, Hilbert curve-Riemannian manifold, Non cooperative game-Nash Inquality-Axiom of determinancy
\end{abstract}

\section{Introduction}

Spin models try hard to capture various complex ferromagnetic phase transition. In solid-state physics, quantum spin hamiltonians describe the effective magnetic interactions between localized electronic spins. As such, they can be derived starting for the full problem of interacting electrons. The Heisenberg exchange is the most important spin-spin interaction and forms the starting point for understanding many materials and phenomena. The dimensionality plays a crucial role in using the correct spin model that will explain the ferromagnetic phase transition in any category of materials. In Ising model, spins are restricted to $\mathrm{z}$ axis but in Heisenberg model the spins are taken in the three ${ }^{*} \mathrm{x}, \mathrm{y}, \mathrm{z}^{*}$ axes. Set theory discusses the problems of diminesionality and cardinality. Brower theory gives the concept of dimensionality its proper position without any separation to the concept of cardinality. Besides, we use it to predict a fractal movement of the magnetic domains. Recently, experiments have verified the fractal movement of the magnetic domains in thin films. Many technological trends use fractal space filling curves to innovate artificial magnetic materials. The paper is organized as follow. In section 2,Residual magnetic field was briefly reviewed.In section 3, Concepts in the theory of set were briefly discussed. In section 4, fractal motion of magnetic domains in thin films was verified experimentally. In section 5, the relation of ferromagnetic models and cantorian set theory was discussed. The work closes with some concluding remark in section 6 .

\section{Residual Magnetic Field}

The difficulty is that including magnetic field without other modification of the Heisenberg Hamiltomain because such result cannot be compared by experiments. Real ferromagnetism at zero applied fields are subdivided into domains which are magnetized in different directions. Pierre - Ernest Weiss suggested existence of magnetic domains in ferromagnets. The most fameous types of domain walls are Bloch wall and Neel wall. Domain walls can be described as topological solitions which occur whenever a discrete symmetry is spontaneous broken (Aharoni, 1995; Coey, 2009; Cullity, 1972).

\section{Concepts in the Theory of Sets}

Set theory is the branch of mathematical logic that studies sets, which informally are collections of objects. Although any type of object can be collected into a set, set theory is applied most often to objects that are relevant to mathematics. The language of set theory can be used in the definitions of nearly all mathematical objects. The modern study of set theory was initiated by Georg Cantor and Richard Dedekind in the 1870s. The cardinality of a set is a measure of the "number of elements of the set. For example, the set $\mathrm{A}=\{2,4,6\}$ 
contains 3 elements, and therefore A has a cardinality of One of Cantor's most important results was that the cardinality of the continuum (2power aleph ) is greater than that of the natural numbers ((2 power aleph ); that is, there are more real numbers $\mathbf{R}$ than whole numbers $\mathbf{N}$. Invariance of domain is a theorem in topology about homeomorphic subsets of Euclidean space $\mathbb{R}^{n}$ (Stillwell, 2013). It states:If $\mathrm{U}$ is an open subset of $\mathbb{R}^{n}$ and f: $\mathrm{U}$ $\rightarrow \mathbb{R}^{n}$ is an injective continuous map, then $\mathrm{V}=\mathrm{f}(\mathrm{U})$ is open and $\mathrm{f}$ is a homeomorphism between $\mathrm{U}$ and $\mathrm{V}$. The theorem and its proof are due to L. E. J. Brouwer, published in 1912. One of the most fameous continuous maps is the space filling curve (Stillwell, 2013; Herrlich, 2009; Kanamori, 2003; Sagan, 2012; Falconer, 2014; Granas $\&$ Dugundji, 2013). We refer to surjective, continuous maps from the unit interval to the unit $n$-cube $(n>1)$ as n-dimensional space-filling curves. Note that we refer to the map(and not its image) as curve. We restrict to this kind of space-filling curves because they are useful for computing orderings of point sets. One of the most fameous space filling curve examples is Hilbert curve.A Hilbert curve is a continuous fractal space-filling curve first described by the German mathematician David Hilbert in 1891 (Sagan, 2012). Fractal Hilbert space filling curve is used to make Artificial Magnetic Materials. The concept of large cardinals and determinancy is so important and we will illustrate it.The era of set theory following the discovery of independence a major concern has been the discovery of new axioms that settle the statements left undecided by the standard axioms (ZFC) (Herrlich, 2009). One interesting feature that has emerged is that there are often deep connections between axioms that spring from entirely different sources. In this section, we will be concerned with one instance of this phenomenon, namely, the connection between axioms of definable determinacy and large cardinal axioms. In the mathematical field of set theory, a large cardinal property is a certain kind of property of transfinite cardinal numbers. Cardinals with such properties are, as the name suggests, generally very "large" (for example, bigger than the least $\alpha$ such that $\alpha=\omega_{\alpha}$ ). The proposition that such cardinals exist cannot be proved in the most common axiomatization of set theory, namely ZFC, and such propositions can be viewed as ways of measuring how "much", beyond ZFC, one needs to assume to be able to prove certain desired results. In other words, they can be seen, in Dana Scott's phrase, as quantifying the fact "that if you want more you have to assume more (Kanamori, 2003). In the mathematical field of set theory, Martin's axiom, introduced by Donald A. Martin and Robert M. Solovay is a statement that is independent of the usual axioms of ZFC set theory. For any cardinal $\mathbf{k}$, we define a statement, denoted by MA(k):For any partial order $P$ satisfying the countable chain condition (and any family $D$ of dense sets in $P$ such that $|D| \leq \mathbf{k}$, there is a filter $F$ on $P$ such that $F \cap d$ is non-empty for every $d$ in $D$. There is a rough convention that results provable from ZFC alone may be stated without hypotheses, but that if the proof requires other assumptions (such as the existence of large cardinals), these should be stated. Whether this is simply a linguistic convention, or something more, is a controversial point among distinct philosophical schools. A large cardinal axiom is an axiom stating that there exists a cardinal (or perhaps many of them) with some specified large cardinal property. Most working set theorists believe that the large cardinal axioms that are currently being considered are consistent with ZFC. These axioms are strong enough to imply the consistency of ZFC. This has the consequence (via Gödel's second incompleteness theorem) that their consistency with ZFC cannot be proven in ZFC (assuming ZFC is consistent). Definition. A cardinal $\kappa$ is a Woodin cardinal if $\kappa$ is strongly inaccessible and for all $\mathrm{A} \subseteq v_{k}$ there is a cardinal $k_{A}<k$ such that $k_{A}$ is $\eta$-A-strong, for each $\eta$ such that $<k_{A} \leq \eta$. $\leq k$ It should be noted that in contrast to measurable and strong cardinals, Woodin cardinals are not characterized as the critical point of an embedding or collection of embeddings. In fact, a Woodin cardinal need not be measurable. However, if $\kappa$ is a Woodin cardinal, then V $\kappa$ is a model of ZFC and from the point of view of $\mathrm{V} \kappa$ there is a proper class of strong cardinalsc (Kanamori, 2003; Koellner \& Woodin, 2010; Shelah \& Woodin, 1990). Going further, a cardinal $\kappa$ is superstrong if there is a transitive class $M$ and a non-trivial elementary embedding $\mathrm{j}: \mathrm{V} \rightarrow \mathrm{M}$ such that crit $(\mathrm{j})=\kappa$ and $\mathrm{V} \mathrm{j}(\kappa) \subseteq \mathrm{M}$. If $\kappa$ is super strong then $k$ is a Woodin cardinal and there are arbitrarily large Woodin cardinals below $\kappa$ (Riis, 1994). One can continue in this vein, demanding greater agreement between $\mathrm{M}$ and $\mathrm{V}$. The ultimate axiom in this direction would, of course, demand that $\mathrm{M}=\mathrm{V}$. This axiom was proposed by Reinhardt. But shortly after its introduction Kunen showed that it is inconsistent with ZFC. In fact, Kunen showed that assuming ZFC, there can be no non-trivial elementary embedding $\mathrm{j}: \mathrm{V} \lambda+2 \rightarrow \mathrm{V} \lambda+2$. (An interesting open question is whether these axioms are inconsistent with ZF or whether there is a hierarchy of "choiceless" large cardinal axioms that climb the hierarchy of consistency strength far beyond what can be reached with ZFC. So, we have two hierarchies of increasingly strong candidates for new axioms and each has a natural limit. Godel's Axiom of Constructibility, V=L provides a conception of the Universe of sets which his perfectly concise modulo only large cardinal axioms which are strong axioms of infinity. However the axiom $V=L$ limits the large cardinal axioms which can hold and so the axiom is false. The Inner Model Program which seeks generalizations which are compatible with large cardinal axioms has been extremely successful, but incremental, and therefore by its very nature unable to yield an ultimate enlargement of $L$. The situation has now changed dramatically and there is, for the first time, a genuine 
prospect for the construction of an ultimate enlargement of $L$ (Koellner \& Woodin, 2010; Shelah \& Woodin, 1990; Gödel, 1939).

\section{Experimental Procedure and Methods \\ 4.1 Methods}

To establish a link between cantorian set theory and ferromagnetic models, fractal motion of magnetic domain must be verified experimentally in 2D lattice. Fractal motion of magnetic domains in different samples of thin films is investigated. All experiments are very fameous. All experiments have been published in public. The paper is in theoretical physics in origin and we support it by fameous experiments.

\subsection{Experimental Data}

50- $\AA \mathrm{Ta} / 25-\AA \mathrm{Pt} /(5-\AA \mathrm{C}$ o90Fe10/10- $\AA \mathrm{Pt}) \mathrm{n}$ films were deposited on a Si substrate with a natural $\mathrm{SiO} 2$ layer via dc-magnetron sputtering and the films were produced with various numbers of repeats $n$ (from 1 to 5 ). Here we chose to use $\mathrm{Co} F$ e films rather than $\mathrm{Co}$ films because $\mathrm{Co}$ Fe films exhibit an almost amorphous phase with small polycrystalline grains. From the magneto-optical Kerr effect measurement, all the films were revealed to exhibit squared out-of-plane hysteresis loops. The coercive fields were revealed to increase monotonically from $113 \mathrm{Oe}$ to 363 Oe as $\mathrm{n}$ increased from 1 to 5 . The films were first saturated under a sufficiently large magnetic field (3,300 Oe) and then, by applying short pulses of reversed magnetic fields (similar strength with the coercive field, 113 Oe, 272 Oe, 295 Oe, and 360 Oe for the samples with $n=1,2,3,4$, and 5, respectively), a few domain nuclei were formed at quenched positions (localized soft magnetic defects). Rather weak magnetic fields, rather than coercive fields, triggered the domain wall to propagate slowly $(10-20 \mu \mathrm{m} / \mathrm{s})$ from the nuclei. The typical domain images are illustrated in Fig1 (Lee, Kim, \& Choe, 2010).
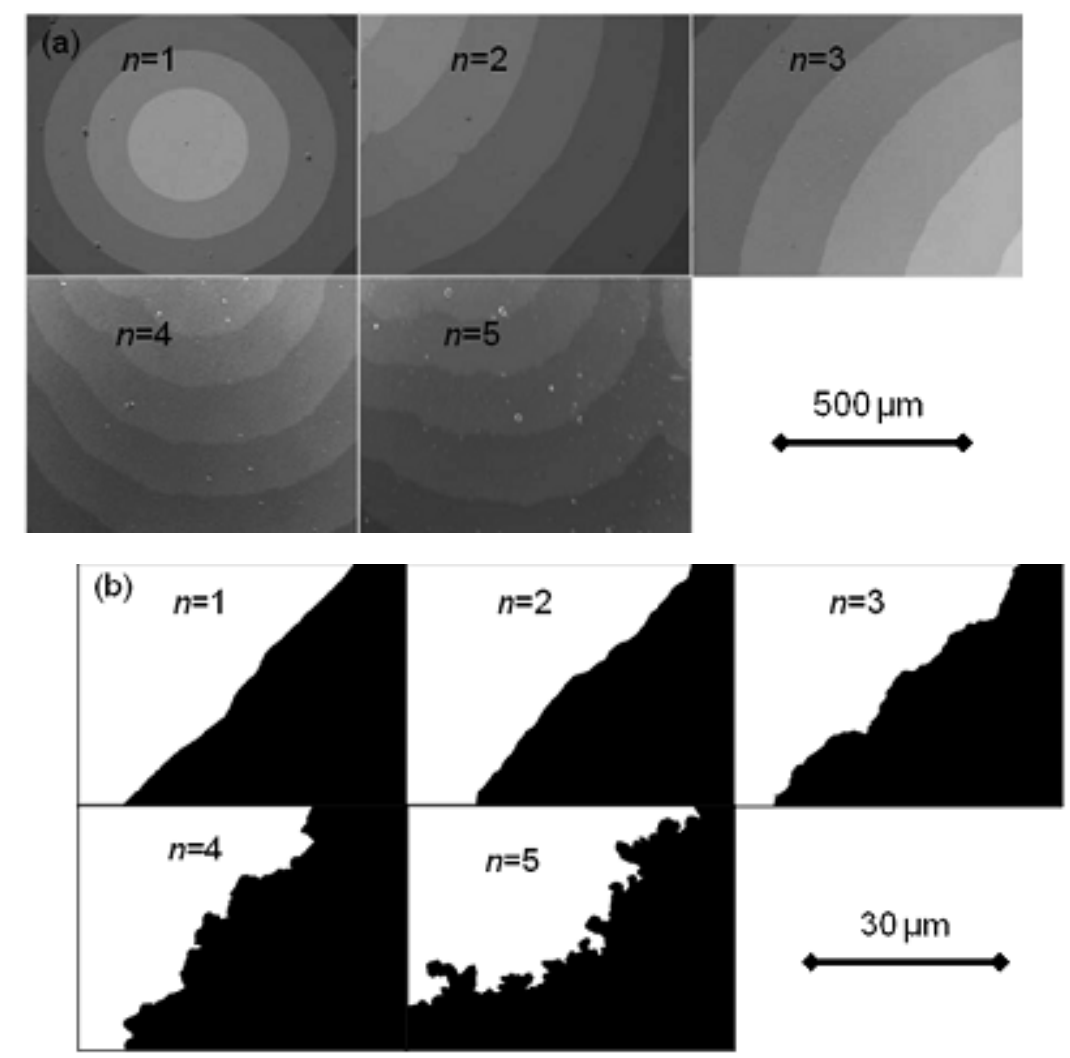

Figure 1. (a) Typical magnetic domain expansion from nucleation sites which are sometimes at the edge of the film with low magnification. The gray constrants correspond to the domain images taken at different times after applying magnetic field. (b) Typical magnetic domain images with high magnification after image processing which comprises background substraction and black and white conversion 
2. A number of $\left(\left(t_{C O} \mathrm{Co} / 11 \mathrm{~A} \mathrm{Pd}\right)\right.$ samples with varying Co Sublayer thickness $t_{C_{o}}$ were prepared on glass substrates by dc magnetron sputtering under $2 \times 10^{-7}$ Torr base pressure. Kerr hysteresis Loop revealing perpendicular magnetic anisotropy.It has been well known that the $\mathrm{Co} / \mathrm{Pd}$ nano multilayer system exhibits an increase of saturation magnetization $M_{z}$ by vibrating sample magenometer. In Figure 1 we demonstrate typical domain evolution paterns $\left(t_{C o} \mathrm{Co} / 11 \mathrm{~A} \mathrm{Pd}\right)$ with $t_{C o}=2,2.5,3,3.5$ and $4 \mathrm{~A}$ observed by MOMM under a constant applied field. it is reported that there is a close relation between fractal geometry and reversal mechanism of magnetic domain in $\mathrm{Co} / \mathrm{Pd}$ nanomultilayers. The fractal Dimension $\mathrm{D}$ of magnetic domain as well as the wall motion speed $\mathrm{V}$, the nucleation rate $\mathrm{R}$, and the reversal ratio $\mathrm{V} / \mathrm{R}$ in $\mathrm{Co} / \mathrm{Pd}$ multinanolayers during magnetization reversal via time resolved direct domain observation. We have carried out direct full field real time observation of magnetic domain for various $\mathrm{Co} / \mathrm{Pd}$ nanomultilayers. The magnetization was triggered by applying a magnetic field in the field range $70-90$ percent of the coercivity to an initially saturated sample. Domain patterns becomes irregular and jagged in case of high demagnetization energy to close magnetic flux at the surface and thus to minimize magnetic energy (Kim, Y. C. Cho, S. B. Choe, \& Shin, 2003).

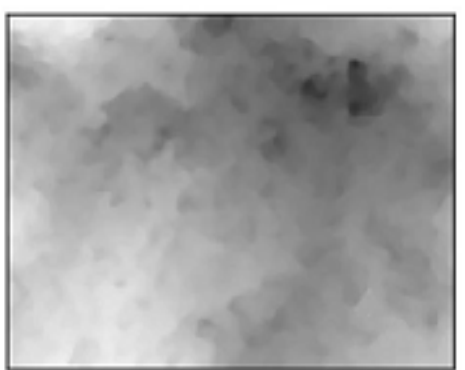

(a)

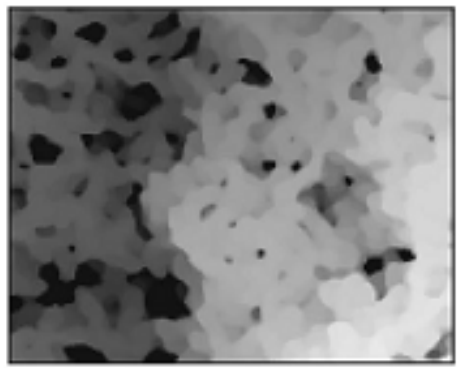

(c)

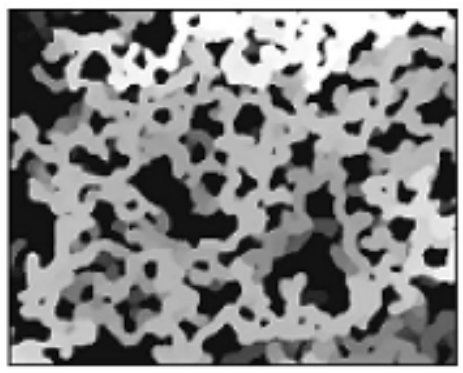

(e)

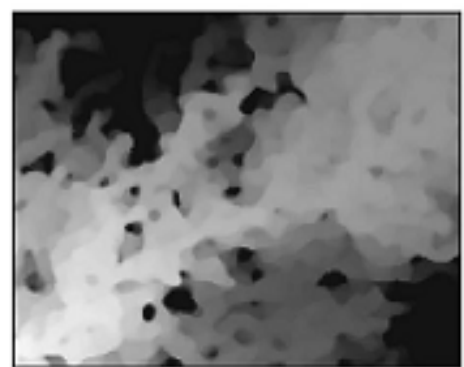

(b)

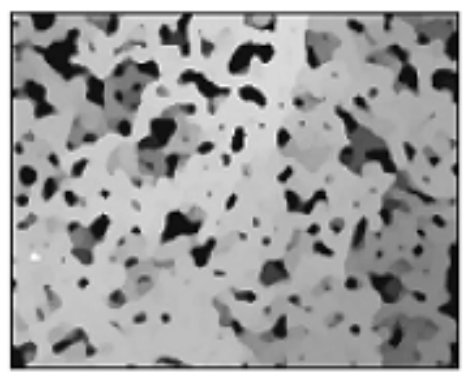

(d)

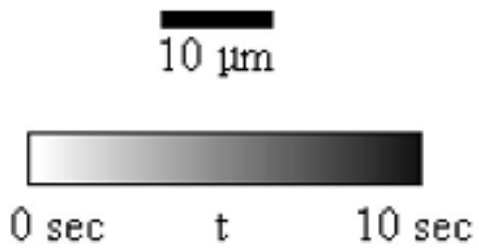

Figure 2. Domain evolution patterns of $\left(t_{C o} A C o / 11 A P d\right) 10$ samples prepared with varying $t_{C_{0}}$ of (a)2.0(b)2.5(c)3.0(d)3.5(e)4 A, respectively. The observed area is $40 \times 32 \mu \mathrm{m}^{2}$ and the gray level represents the elapsed time from 0 to 10 seconds in the process of domain evolution

3. Self-assembly of fractal nano wires and stripe magnetic domain on stretchable substrate has magnetic properties of Ni thin films on polydimethylsiloxane (PDMS) are being studied. Coercivity of the films grown on PDMS is two to three times higher than their rigid counterpart. Magnetic force microscopy studies show that surface morphology and magnetic domains are quite different on elastic substrate. $5 \mathrm{~nm} 5 \mathrm{~nm}$ thick films do not 
exhibit planar morphology but rather form very long nanowires. The 50nm50nm thick films exhibit in-plane and canted magnetizations with nonmagnetic disordered regions within the thin films (Yousefi \& Ramahi, 2010).

4. Probing Fractal Magnetic Domains on Multiple Length Scales in Nd2Fe14BThe industrial strength ferromagnet, Nd2Fe14B has become a prototypical system for the study of magnetic domain structures. Below the Curie temperature, $\mathrm{Tc}^{1} / 4565 \mathrm{~K}$, the Nd and $\mathrm{Fe}$ moments order ferromagnetically. The crystal-electric field produces a strong magnetic anisotropy with the easy axis along the tetragonal $\mathrm{c}$ direction. Below the spin-reorientation temperature, TSR 1/4 $135 \mathrm{~K}$, the magnetic structure (and easy axis direction) changes via a second order transition and becomes cone like, in which the moments are canted away from the c direction by an angle that increases from 0 at T SR to 28 at $4 \mathrm{~K}$. The moments lie in one of the four symmetry-equivalent f1 10 $\mathrm{g}$ planes in agreement with calculations of crystal-electric field effects. A multitude of techniques have been used to image magnetic domains at exposed surfaces of Nd2Fe14B, such as Bitter decoration, Kerr microscopy, Lorentz, and holographic transmission electron microscopy, scanning electron microscopy, and magnetic force microscopy. For imaged surfaces perpendicular to the $\mathrm{c}$ direction, domains have been observed with dimensions between $2-5 \mathrm{~m}$ at $4 \mathrm{~K}$ and about $0: 1-0: 6 \mathrm{~m}$ at room temperature], respectively. These values are very close to the single domain size of about $0: 2-0: 4 \mathrm{~m}$ determined by magnetization measurements on polycrystalline samples manifest smooth, mirrored surfaces and can have volumes as large as $1 \mathrm{~cm} 3$ (Kreyssig et al., 2009).

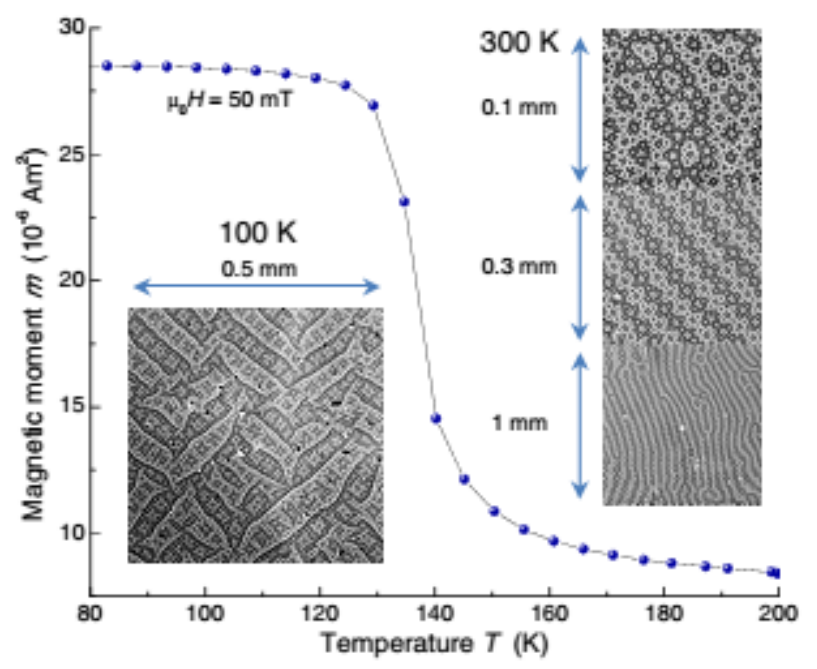

Figure 3. Temperature denendence of magnetization and the magnetic domain natterns Nd2Fe14B single crystal. The magnetization was measured at $\mu_{0} H=50 \mathrm{mT}$ applied along $\mathrm{C}$ axis

5. Novel configurations based on Fractal Hilbert curves are proposed for realizing artificial magnetic materials. It is shown that the proposed configuration gives significant rise to miniaturization of artificial unit cells which in turn results in higher homogeneity in the material, and reduction in the profile of the artificial substrate. Analytical formulas are proposed for design and optimization of the presented structures, and are verified through full wave numerical characterization. The electromagnetic properties of the proposed structures are studied in detail and compared to square spiral from the point of view of size reduction, maximum value of the resultant permeability, magnetic loss, and frequency dispersion. To validate the analytical model and the numerical simulation results, an artificial substrate containing second-order Fractal Hilbert curve is fabricated and experimentally characterized using a microstrip-based characterization method (Aurongzeb, 2006). 


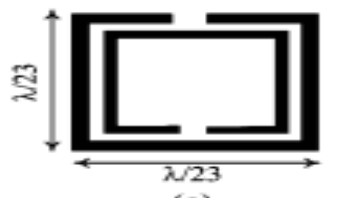

(a)

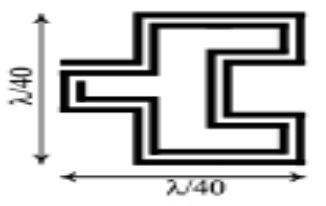

(c)

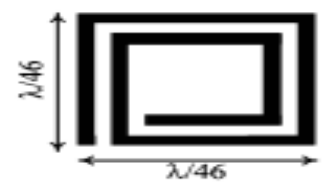

(b)

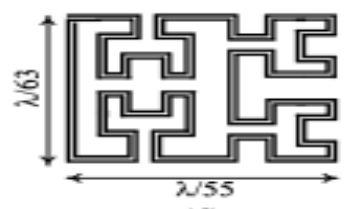

(d)

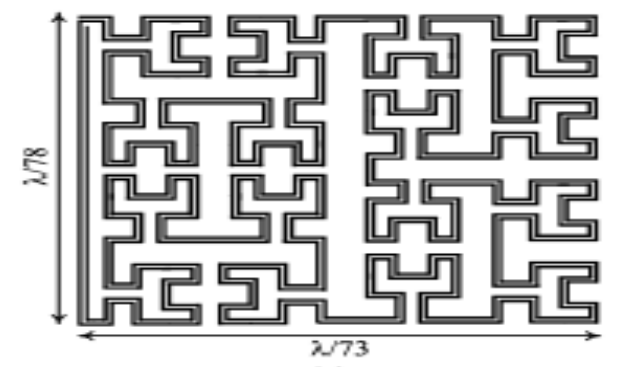

Figure 4. (a)SRR(b)Square Spiral (c)Second order Fractal Hilbert Inclusion(d) Third order Fractal Hilbert Inclusion(e) Fourth order Fractal Hilbert Inclusion. Note that as the order of Hilbert Curve increases, the size of inclusion decreases.

6. Fractals are promising candidates as nonperiodic, nonresonant structures exhibiting a homogeneous, isotropic, and frequency-independent effective optical response. We present a comprehensive optical investigation of a metallic Hilbert curve of fractal order $N=9$ in the visible and near-infrared spectral range. experiments show that high-order fractal nanostructures exhibit a nearly frequency independent reflectance and an in-plane isotropic optical response (De Zuani et al., 2015).
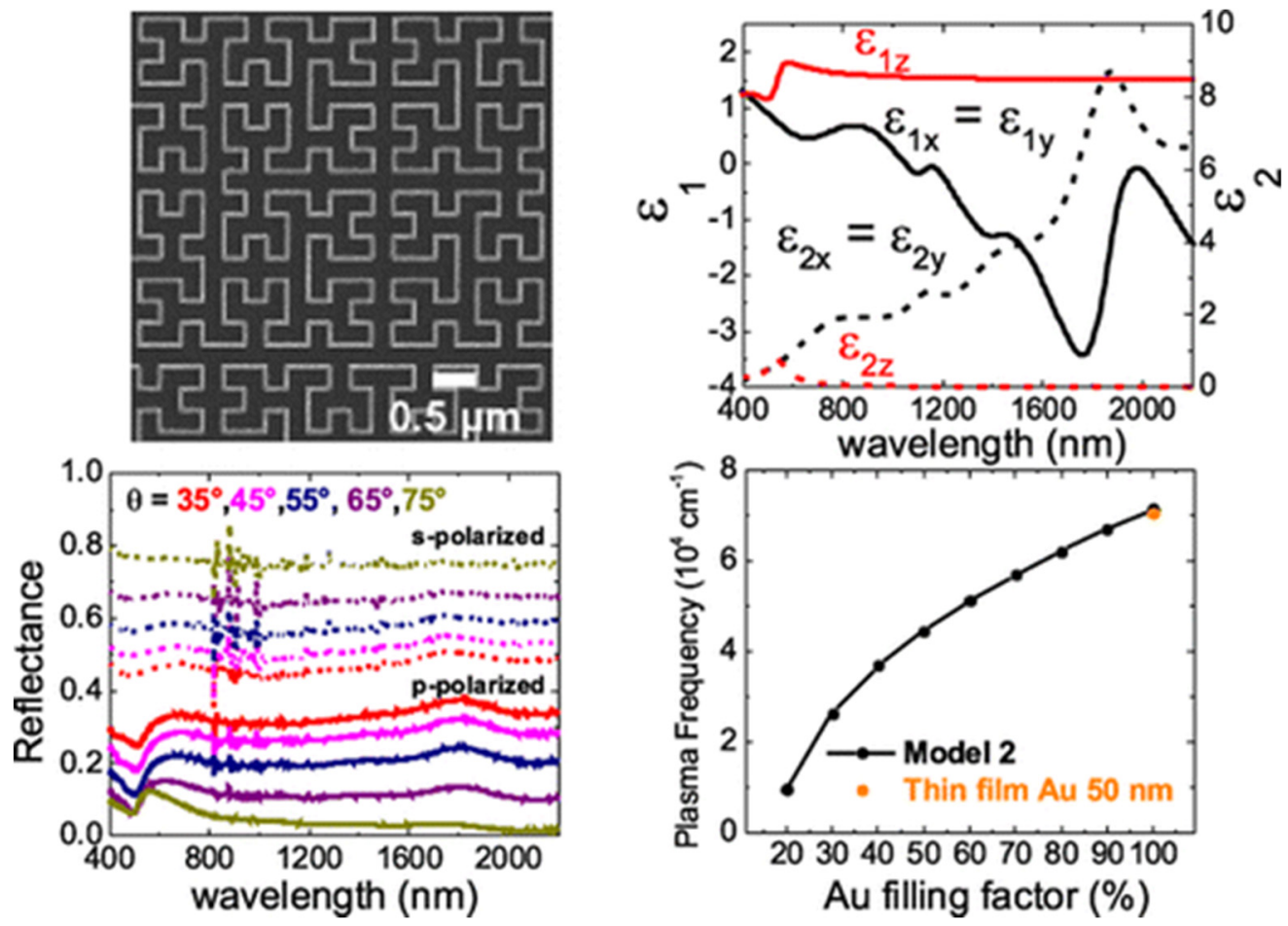

Figure 5. Optical investigation of metallic Hilbert Curve of fractal order $\mathrm{N}=9$ 


\section{Results and Discussions}

1. After applying and removing magnetic field $\mathrm{H}=0$, we can assume that there are magnetic domains in $\mathrm{XYZ}$ directions and these domains can be written as:

$\left[a_{1}, b_{1}\right] \times\left[a_{2}, b_{2}\right] \times\left[a_{3}, b_{3}\right] \subset \mathbb{R}^{3}$ or they will be in $\mathrm{XY}$ directions and can be written as:

$\left[a_{1}, b_{1}\right] \times\left[a_{2}, b_{2}\right] \subset \mathbb{R}^{2}$

2. These domains have the cardinality 2 power aleph

3. We will use the theory of invariance of dimensions which is proved by Brouwer as the domains $\left[a_{1}, b_{1}\right]$ $\times\left[a_{2}, b_{2}\right] \times\left[a_{3}, b_{3}\right] \subset \mathbb{R}^{3}$ or, $\left[a_{1}, b_{1}\right] \times\left[a_{2}, b_{2}\right] \subset \mathbb{R}^{2}$ have 2 power aleph cardinality.

4. Using Brouwer theory, there will be no bijection if magnetic domains $\left[a_{1}, b_{1}\right] \times\left[a_{2}, b_{2}\right] \times\left[a_{3}, b_{3}\right] \subset \mathbb{R}^{3}$ are transported to $\mathrm{z}-$ axis.

5. The reason of restriction of the spins of Ising model in $\mathrm{z}$ axis is that there is $T: H_{X Y Z} \rightarrow H_{Z}$ (continous or injective). But in the case of Heisenberg Hamiltonian there will be no restriction as there is no function $T: H_{x y z} \rightarrow H_{z}$

6. In the case of materials that is explained by Ising model, temperature will have the concept of the function that transport magnetic domain to $\mathrm{z}$ - axis, $T: H_{X Y Z} \rightarrow H_{Z}$

7. In phase transitions that are explained only by Ising model, we can expect fractal behavior in general in these materials.

8. These fractal motions belong to space - filling curve category ( Peano, Hilbert, ..... etc ) curve will explain it.

9. Space filling curves like Hilbert Space are continous functions will appear in Ising model materials as $T: H_{X Y Z} \rightarrow H_{Z}$ is continous.

11. Heisenberg model shows the spins in $\left(\mathrm{Sx}, \mathrm{S}_{\mathrm{y}}, \mathrm{S}_{\mathrm{z}}\right)$, so there will be no fractal behavior in the material that exhibit phase transitions under it.

13. The phase transitions of thin films are exhibited by Ising model mainly and they cannot be explained by Heisenberg Model.

14.Summary of magnetic model, where d/D (dimension of spins /dimension of lattice)

\begin{tabular}{|c|c|c|c|}
\hline $\mathrm{d} / \mathrm{D}$ & 1 & 2 & 3 \\
\hline 1. I sing & $\times$ & $\sqrt{ }$ & $\sqrt{ }$ \\
\hline 2. (XY) & $\times$ & $\sqrt{ }$ & $\sqrt{ }$ \\
\hline 3. (Heisenberg) & $\times$ & $\times$ & $\sqrt{ }$ \\
\hline
\end{tabular}

15. We can say Ising Model can explain thin films but Heisenberg Model cannot explain thin films. Spin flucations are strongly dependent on temperature, so we can consider it as a tool to describe the phase transitions.

The assumption of the existence of magnetic domain with cardinality ( 2 power aleph) solve the problem of dimensionality of the spin models and give the proper explanation to the difference between Heisenberg model and Ising model.

We consider the case of materials that is explained by Ising model, temperature will have the concept of the function that transport magnetic domain to $\mathrm{z}$ - axis, $T: H_{X Y Z} \rightarrow H_{Z}$, so we can expect fractal motion in these materials. The fractal properties of spin clusters and boundries which are cluster themselves are described by percolation theory. Asymptotically, cluster distributions take a general form:

$$
l_{n} \sim n^{-\tau} \exp (-\theta n)
$$

Fortuin and Kasteleyen showed that spin clusters can be constructed for the potts model that do percolates at $T_{C}$ and encode the thermal critical behavior. The percolation thresholds of both FK and geometrical clusters 
concides with thermal critical temperature of 2D Ising model. The entropy exponent $\tau$ determines the fractal structures of geometrical objects (Janke \& Schakel, 2005).

The previous assumptions are agreement with fractional Ginzburg-Landau equations for fractal media.. As we have assumed temperature is a continuous function to express about the fractal motion of magnetic domains. Free energy is an important thermodynamical tool to describe the behavior of any system. Ginzburg -landau equations use this term to describe the motion in fractal media. Let us consider the thermodynamic potential (free energy functional) $\mathrm{F}\{\mathrm{Z}(\mathrm{x})\}$ for the non equilibrium state of the fractal medium, where $\mathrm{Z}(\mathrm{x})$ is a scalar field. The free energy functional has the form (Ginzburg \& Landau, 1950).

$$
F\{Z(x)\}=F_{0}+\int_{W} \mathrm{~F}(Z(x), \nabla Z(x)) d V_{3}
$$

where $F(Z(x), \nabla Z(x))$ is the free energy density; $\nabla Z=\partial Z / \partial x$. For the Ginzburg-Landau potential, this density is $\mathrm{F}(\mathrm{Z}(\mathrm{x}), \nabla \mathrm{Z}(\mathrm{x}))=1$. Here $\mathrm{D}$ is a fractal mass dimension of the fractal medium, and $d V_{D}$ is an element of the D-dimensional volume:

$$
d V_{D}=C_{3}(D, x) d V_{3}
$$

The location of the transition is a function of the temperature, field amplitude and frequency. A finite-size scaling analysis of large-scale Monte Carlo simulations of the kinetic Ising model in an oscillatory field has shown that the dynamic phase transition is in the same universality class as the equilibrium Ising model. A result confirmed in a recent study of a time-dependent Ginzburg-Landau model in an oscillatory field. The dynamic response of thin ferromagnetic Heisenberg films with competing surface fields to an applied oscillatory field has been studied by Hyunbum Jang1 and Malcolm J Grimson. The magnetic spins in the model are continuously orientable, but the bilinear exchange anisotropy $\Lambda$ in the Heisenberg Hamiltonian ensures that Ising-like characteristics are retained. The competition between the ferromagnetic ordering tendencies of the spins and the applied oscillatory field determines the behavior of the film, which exhibits a dynamic phase transition between dynamically ordered and dynamically disordered phases. The critical temperature of the dynamic phase transition, Tcd, is a function of the angular frequency $\omega$ and amplitude H0 of the applied oscillatory field. Hysteresis loops centered on the origin are observed at temperatures above Tcd. But for $\mathrm{T}<\mathrm{Tcd}$ the film is in a dynamically ordered state and the hysteresis loop is displaced from the origin being located in either the positive or negative magnetization half plane depending on the initial conditions of the simulation. A study of the time-dependent layer magnetization across the film $\operatorname{Mnz}(t)$, has shown that for $\mathrm{T}<\mathrm{Tcd}$ the dynamically ordered and dynamically disordered phases coexist within the film. Monte Carlo algorithm was the Metropolis method.

Simulations were performed on the HITAC S810 model 20 at the Computer Center of University of Tokyo. In two-dimensional case, we have studied not only at the critical point, but also at other temperatures around the critical point. The system studied here was the 64 x 64 square lattice Ising model with cyclic boundary conditions in all directions. showed in Fig 6 (De Zuani et al., 2015; Akınc1, 2014; Jang \& Grimson, 2001; Huang, Zhang, Chen, \& Du, 2005; Ito \& Suzuki, 1987).

Critical properties of the Ising models on some two dimensional deterministic Sperinski fractals with different Haussdorff measures. Haussdorff measures are strongly related with the axiom of martin (Carmona et al., 1998), Theorem.5.1: Let $s \geq 0$ and let $H^{s}$ be the s-dimensional Haussdorff measure in $\mathbb{R}^{n}$.The union of less than continuum many $H^{s}$ negligible subsets of $\mathbb{R}^{n}$ is $H^{s}$ negligible (Davies \& Rogers, 1969; Fremlin, 1984).

Søren Riis showed by using the axiom of choice, the construction of a symmetrical and self similar subset $A \subseteq[0,1] \subseteq \mathrm{R}$. Then by an elementary strategy stealing argument it is shown that $A$ is not determined. The (possible) existence of fractals like $A$ clarifies the status of the controversial Axiom of Determinacy (Riis, 1994; Myceilsky, 1964; Shelah \& Woodin, 1990; Fremlin, 1984). His beautiful research showed there is a fractal that violates the axiom of determinancy. In section 3 , we have showed the relation between large cardinals, the axiom of martin and the axiom of determinancy in section 3. So can we enlarge our model to enter the concept of large cardinals? Recently, there is no experimental data but this concept must be studied experimentally. 

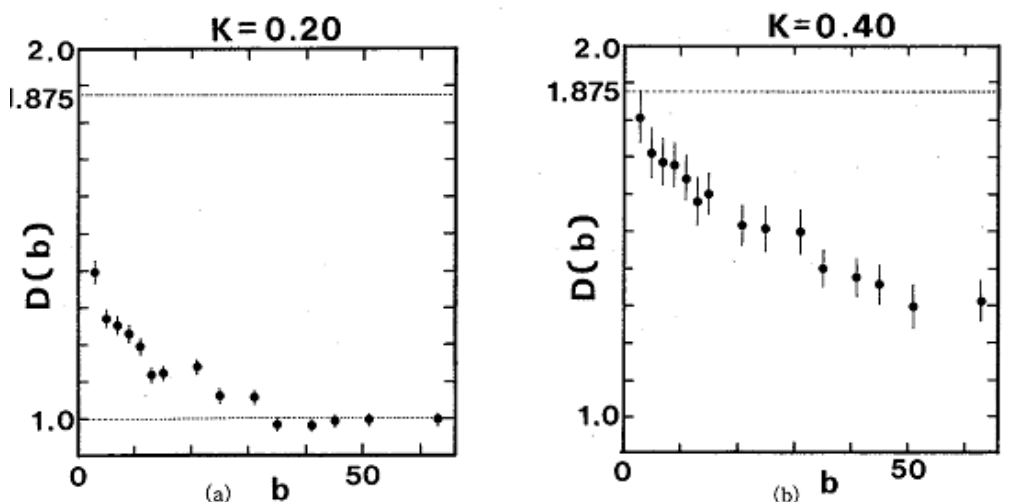

$K=0.43$
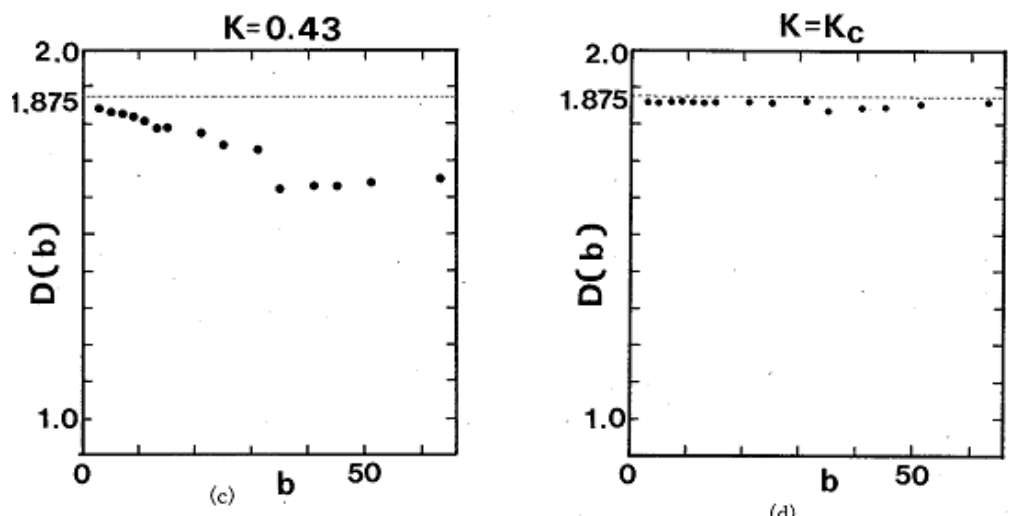

Figure 6. Values $\mathrm{D}(\mathrm{b})$ defined by equation(3) are shown as function of scale factor $\mathrm{b}$ for several temperatures. The system size $64 \times 64(\mathrm{a}) \mathrm{K}=.2$ when the sacle $\mathrm{b}$ is large the values $\mathrm{D}(\mathrm{b})$ approaches to unity, which corresponds to random percolation case. $(\mathrm{b}) \mathrm{K}=.4(\mathrm{c}) \mathrm{K}=.43(\mathrm{~d}) \mathrm{K}=\mathrm{kc}=.4407 \mathrm{D}(\mathrm{b})$ converges to the expected value 1.875 irrespective of the used scale factor (Ito \& Suzuki, 1987)

It has been showed the vortex solutions of the Landau Lifshits model of ferromagnetism on $\mathbb{R}^{2}$ with an easy axis anisotropy. There is a tendancy to the fractal motion in Landau Lifshits model (Henriksen, 1953; Piette \& Zakrzewski, 1998; Ding \& Wang, 1998).

Magnetic bubbles is a small round magnetic domain induced by magnetic field in a thin film of magnetic material. Let us investigate solutions in a 2 dimensional Landau- Lifshits model. We look at Landau -Lifshits model for the anistropic Heisenberg model

$$
\partial_{t} \phi=\phi\left[\nabla^{2} \phi+A(\phi . n) n\right]
$$

where $\phi$ is a unit vector describing the orientation of magnetization and $n$ is the vector of external magnetic field, the total energy of the system:

$$
E=1 / 8 \int d x d y\left[\left(\partial_{x} \phi, \partial_{y} \phi\right)+\left(\partial_{y} \phi, \partial_{x} \phi\right)+A\left(1-\phi^{2}\right)\right]
$$

where $\mathrm{A}$ is a positive constant, Instead of the normalized vector $\phi$ to describe the magnetization it is convenient to perform stereographic projection of the sphere $\omega=\left(\varphi_{1}+i \varphi_{2}\right) /\left(1+\varphi_{3}\right)$. Finally, the equation of motion

$$
i \omega_{t}+\omega_{x x}+\omega_{y y}-\left(2 \omega^{*}\left(\omega_{x}^{2}+\omega_{y}^{2}\right) / 1+(\omega)^{2}\right)-A \omega\left(1-\omega^{2} / 1+\omega^{2}\right)=0
$$

when the external magnetic field is switched off $\mathrm{A}=0$, the static solution is given by holomorphic functions (Jiang, 1953). Singular manifolds can be well-defined in a framework that is, in fact, quite natural, and emerges quite often in theoretical physics, namely the holonomic functions (Piette \& Zakrzewski, 1998) corresponding to $\mathrm{n}$-fold integrals of a holonomic integrand (most of the time, in theoretical physics, the integrand is simply rational or algebraic). In Sato's Dmodule theory (Ding \& Wang, 1998), a holonomic system is a highly 
over-determined system, such that the solutions locally form a vector space of finite dimension (instead of the expected dependence on some arbitrary functions). Furthermore, holonomic functions naturally correspond to systems with fixed regular singularities. It is crucial to avoid movable singularities. For non-holonomic functions, only the ones that can be decomposed as an infinite sum of holonomic functions (like $\chi$, the full susceptibility of the square $\dagger$ Ising model (Meyer, 1999; Eisert, Wilkens, \& Lewenstein, 1999)) give some hope for interesting and/or rigorous studies of their singularities. Let $\mathrm{M}$ be a connected $\mathbb{C}$ manifold, Kapovich proved the striking result :if $\mathrm{M}$ has a non constant holomorphic function then the ring of holomorpbhic function on $\mathrm{M}$ has a chain of prime ideals of the length of continuum(2 power aleph) (Carmona et al., 1998; Sasane, 2008). C.F. Lee and Neil Johnson explore the possibility that physical phenomena arising from interacting multi-particle systems, can be usefully interpreted in termsof multi-player games. We show how non-cooperative phenomena can emerge from Ising Hamiltonians, even though the individual spins be- have cooperatively. Our findings establish a mapping between two fundamental models from condensed matter physics and game theory. So, if we have the ability to design a magnetic material respected to the fractal which violates the axiom of determinancy that is showed by soren riis, there will be fantastic results to understand many aspects of ferromagnetism.

There are many aspects for solobev space in the solution of Ising model, so let us remind Nash Inquality on connected Riemannian manifold where heat kernels are well defined. Very often (and we shall see that Nash inequalities provide a useful criterium for this), this kernel has a density with respect to the reversible measure $\mu$, that is $\operatorname{Pt}(\mathrm{x}, \mathrm{dy})=\operatorname{pt}(\mathrm{x}, \mathrm{y}) \mu(\mathrm{dy})$; here $\mathrm{pt}(\mathrm{x}, \mathrm{y})$ is a non negative function which is defined almost everywhere (with respect to $\mu \otimes \mu$ ) on $\mathrm{E} \times \mathrm{E}$. Then the symmetry property (iv) is equivalent to thesymmetry of this kernel $\operatorname{pt}(x, y)=\operatorname{pt}(y, x)$. Much attention has been brought over the last decades to various estimates on this kernel density (in particular in Riemannian geometry, for heat kernels on Riemannian manifolds, using tools from geometry like curvature, Riemannian distance, etc). Once again, Nash inequalities may provide good such estimates, as we shall see later on.

The knowledge of the measure and of the carré du champ (or of the Dirichlet form) entirely describes the operator L (and therefore the semigroup), since L may be definedfrom $\square$ and $\mu$ through the integration by parts formula (see (1.3)). The basic example of such semigroups is of course the standard heat kernel in the Euclidean space $\mathrm{Rn}$; for $\mathrm{t}>0$, its density $\mathrm{pt}(\mathrm{x}, \mathrm{y})$ with respect to the Lebesgue measure dy is $p_{t}(x, y)=\frac{1}{(4 \pi t)^{n / 2}} \exp \left(-\frac{|x-y|^{2}}{4 t}\right)$, Here, $\mu(\mathrm{dy})=\mathrm{dy}, \mathrm{L}$ This corresponds to the case studied by Nash (Carlen \&

Loss, 1993; Davies, 1990).

\section{Conclusion}

The relation between ferromagnetic models and cantorian set theory has been studied. New model has been proposed and has been compared by Landau_Ginzburg equations. The fractal motion of magnetic domain has been predicted theoretically and verified experimentally. The relation between large cardinals and the axiom of determinancy has been illustrated. Recently, the axiom of determinancy is violated by a special kind of fractal. So, can we investigate ferromagnetic models under the scope of large cardinals by an experimental evidence? or can we design a magnetic material under the scope of large cardinals?

\section{References}

Aharoni, A. (1995). Introduction to the theory of ferromagnetism (International Series of Monographs in Physics, pp. 60-80). Oxford Science Publication. http://dx.doi.org/10.1063/1.881916

Akıncı, Ü. (2014). Anisotropic Heisenberg model in thin film geometry. Thin Solid Films, 550, 602-607. http://dx.doi.org/10.1016/j.tsf.2013.10.002

Aurongzeb, D. (2006). Self-assembly of fractal nanowires and stripe magnetic domain on stretchable substrate. Applied physics letters, 89(12), 123128. http://dx.doi.org/10.1063/1.2353825

Bathany, C., Le Romancer, M., Armstrong, J. N., \& Chopra, H. D. (2010). Morphogenesis of maze-like magnetic domains. Physical Review B, 82(18), 184411. http://dx.doi.org/10.1103/PhysRevB.82.184411

Bejenaru, I., Ionescu, A. D., Kenig, C. E., \& Tataru, D. (2011). Global Schrödinger maps in dimensions $\$ \mathrm{~d} \geq 2 \$$ : Small data in the critical Sobolev spaces. Annals of Mathematics, 173(3), 1443-1506. http://dx.doi.org/10. 4007/annals.2011.173.3.5 
Carlen, E. A., \& Loss, M. (1993). Sharp constant in Nash's inequality. International Mathematics Research Notices, 1993(7), 213-215.

Carmona, J. M., Marconi, U. M. B., Ruiz-Lorenzo, J. J., \& Tarancón, A. (1998). Critical properties of the Ising model on Sierpinski fractals: A finite-size scaling-analysis approach. Physical Review B, 58(21), 14387. http://dx.doi.org/10.113/PhysRevB58.14387

Coey, J. M. D. (2009). Magnetism and Magnetic Materials (pp. 128-174). Cambridge University Press. http://dx.doi.org/10.1017/CBO9780511845000

Cullity, B. D. (1972). Introduction to Magnetic Materials (pp. 275-300). Addison-Wesley, London. http://dx.doi.org/10.1002/9780470386323

Davies, E. B. (1990). Heat kernels and spectral theory. Cambridge Tracts in Mathematics (volume 92). Cambridge University Press.

Davies, R. O., \& Rogers, C. A. (1969). The problem of subsets of finite positive measure. Bulletin of the London Mathematical Society, 1(1), 47-54. http://dx.doi.org/10.1112/blms/1.1.47

De Zuani, S., Reindl, T., Rommel, M., Gompf, B., Berrier, A., \& Dressel, M. (2015). High-Order Hilbert Curves: Fractal Structures with Isotropic, Tailorable Optical Properties. ACS Photonics, 2(12), 1719-1724. http://dx.doi.org/10.1021/acsphotonics.5b00363

Ding, W., \& Wang, Y. (1998). Schrödinger flow of maps into symplectic manifolds. Science in China Series A: Mathematics, 4l(7), 746-755. http://dx.doi.org/10.1007/BF02901957

Eisert, J., Wilkens, M., \& Lewenstein, M. (1999). Quantum games and quantum strategies. Physical Review Letters, 83(15), 3077.

Falconer, K. (2014). Fractal Geometry: Mathematical Foundations and Applications (pp44-66). Willey. http://dx.doi.org/10.1002/0470013850

Fan, F. (n.d.). In H. W, Kuhn, \& A. W. Tucker (Eds.), Annals of Mathematics Studies 38 (p.99). Princeton Press.

Fremlin, D. H. (1984). Consequences of Martin axiom (pp. 1-15). Cambridge university press, Cambridge.

Ginzburg, V. L., \& Landau, L. D. (1950). Zh. Eksp. Teor. Fiz. 20, 1064. In L. D. Landau (Trans.). Oxford: Pergamon Press, 1965. http://dx.doi.org/10.1103/PhysRevD.76.041701

Gödel, K. (1939). Consistency-proof for the generalized continuum-hypothesis. Proceedings of the National Academy of Sciences, 25(4), 220-224. http://dx.doi.org/10.1007/BF01700692.

Granas, A., \& Dugundji, J. (2013). Fixed point theory (pp9-35). Springer Science \& Business Media. http://dx.doi.org/10.1007/978-0-387-21593-8.

Gross, L. (1975). Logarithmic Sobolev inequalities. Amer. J. Math., 97(4), 1061-1083.

Hart, M. L., \& Johnson, N. F. (2002). Crowd-anticrowd theory of multi-agent minority games. arXiv preprint cond-mat/0212088.

Henriksen, M. (1953). On the prime ideals of the ring of entire functions. Pacific J. Math, 3, 711-720. http://dx.doi.org/10.2140/pjm.1953.3.711.

Herrlich, H. (2009). Axiom of Choice. Mathematics, 1876, 20-30. http://dx.doi.org/10.1007/11601562

Huang, Z., Zhang, F., Chen, Z., \& Du, Y. (2005). Dynamic transition and hysteresis scaling in Heisenberg ferromagnet. The European Physical Journal B-Condensed Matter and Complex Systems, 44(4), 423-430. http://dx.doi.org/10.1140/epjb/e2005-00141-4

Ito, N., \& Suzuki, M. (1987). Fractal configurations of the two-and three-dimensional Ising models at the critical point. Progress of theoretical physics, 77(6), 1391-1401.

Jang, H., \& Grimson, M. J. (2001). Hysteresis and the dynamic phase transition in thin ferromagnetic films. Physical Review E, 63(6), 066119. http://dx.doi.org/10.1103/PhysRevE.63.066119

Janke, W., \& Schakel, A. M. (2005). Fractal structure of spin clusters and domain walls in the two-dimensional Ising model. Physical Review E, 71(3), 036703. http://dx.doi.org/10.1103/PhysRevE.71.036703

Jiang, R. Q. (1953). On Vortex solutions of the Landau Lifshits equations. Pacific J. Math., 3, 711-720. http://dx.doi.org/10.2140/pjm.1953.3.711. 
Kanamori, A. (2003). The higher infinite: large cardinals in set theory from their beginnings. Springer Science \& Business Media. http://dx.doi.org/10.2307/2275615

Kim, D. H., Cho, Y. C., Choe, S. B., \& Shin, S. C. (2003). Correlation between fractal dimension and reversal behavior of magnetic domain in Co/Pd nanomultilayers. Applied physics letters, 82(21), 3698-3700. http://dx.doi.org/10.1063/1.1578185

Koellner, P., \& Woodin, W. H. (2010). Large cardinals from determinacy. In Handbook of set theory (pp. 1951-2119). Springer Netherlands. http://dx.doi.org/10.1007/978-1-4020-5764-9_24

Kreyssig, A., Prozorov, R., Dewhurst, C. D., Canfield, P. C., McCallum, R. W., \& Goldman, A. I. (2009). Probing fractal magnetic domains on multiple length scales in Nd 2 Fe 14 B. Physical review letters, 102(4), 047204.

Le Calvez, P. (2006). From Brouwer theory to the study of homeomorphisms of surfaces. In International Congress of Mathematicians (Vol. 3, pp. 77-98). http://dx.doi.org/10.4171/022-3/4

Lee, C. F., \& Johnson, N. F. (2002). For a simple inroduction to games in the quantum domain. Physics World, $15(10), 25$.

Lee, J. M. (1997). Riemannian Manifolds: An Introduction to Curvature (Graduate Texts in Mathematics). Springer. http://dx.doi.org/10.1007/b98852

Lee, K. S., Kim, D. H., \& Choe, S. B. (2010). Fractal Dimension of Magnetic Domain Walls in CoFe/Pt Multilayers. Journal of Magnetics, 15(3), 99-102. http://dx.doi.org/10.4283/JMAG.2010.15.3.099

Li, Y., \& Wang, Y. (2006). Bubbling Location for F harmonic maps and inhomogeneous Landau Lifshits equations. Math Helv, 81(2006), 433-448. http://dx.doi.org/10.4171/CMH/57.

Meyer, D. A. (1999). Quantum strategies. Physical Review Letters, 82(5), 1052.

Myceilsky, J. (1964). On the axiom of determinateness Fund. Math. 54(1964), 05-224. http://dx.doi.org/10.2307/ 2272655

Nash, J. (1958). Continuity of solutions of parabolic and elliptic equations. American Journal of Mathematics, 80(4), 931-954.

Osborne, M. J., \& Rubinstein, A. (1994). A course in game theory. MIT press.

Piette, B. M. A. G., \& Zakrzewski, W. J. (1998). Localized solutions in a two-dimensional Landau-Lifshitz model. Physica D: Nonlinear Phenomena, 119(3), 314-326. http://dx.doi.org/10.1016/S0167-2789(98)00084-0

Riis, S. (1994). Fractal which violates the axiom of choice (Basic Research in Computer Science). http://dx.doi.org/10.7146/brics.v1i24.21642.

Robert, C. H. (2000). Modern magnetic materials: principles and applications. DOI: 10.1109/MEI.2005.1490004

Sagan, H. (2012). Space-filling curves. Springer Science \& Business Media (pp. 50-70). http://dx.doi.org/10. 1007/978-1 -4612-0871-6

Sasane, A. (2008). On the Krull dimension of rings of transfer functions. Acta Applicandae Mathematicae, 103(2), 161-168. http://dx.doi.org/10.1007/s10440-008-9227-1.

Shelah, S., \& Woodin, H. (1990). Large cardinals imply that every reasonably definable set of reals is Lebesgue measurable. Israel Journal of Mathematics, 70(3), 381-394. doi:10.2178/bsl/1182353934

Stillwell, J. (2013). The real numbers (pp. 57-80). Springer International Publishing. http://dx.doi.org/10.1007/ 978-3-319-01577-4

Turner, P. E., \& Chao, L. (1999). Prisoner's dilemma in an RNA virus. Nature, 398(6726), 441-443.

Weibull, J. W. (1997). Evolutionary game theory. MIT press.

Yousefi, L., \& Ramahi, O. M. (2010). Artificial magnetic materials using fractal Hilbert curves. IEEE Transactions on Antennas and Propagation, 58(8), 2614-2622. http://dx.doi.org/10.1109/TAP.2010.2050438

\section{Copyrights}

Copyright for this article is retained by the author(s), with first publication rights granted to the journal.

This is an open-access article distributed under the terms and conditions of the Creative Commons Attribution license (http://creativecommons.org/licenses/by/4.0/). 